\title{
Quasisymmetric Stellarators
}

\author{
Magnetic-field configurations that improve confinement of fusion plasmas \\ in stellarators can be achieved more precisely than previously thought, \\ according to a numerical study.
}

By Linjin Zheng

$\mathrm{H}$ arnessing controlled nuclear fusion as an energy source promises a safe, long-term solution to the twin problems of rising global energy demand and increasing levels of atmospheric carbon dioxide. But in order to generate energy from fusion sustainably, researchers need to find a way to confine very-high-temperature plasmas for long periods. Most reactor designs seek to achieve this goal primarily using magnetic fields, with the front-runner being a simple tokamak geometry in which the plasma circulates within a donut-shaped chamber. The International Thermonuclear Experimental Reactor, for example, which is currently under construction, employs such a design [1] (see Q\&A: A 4D Fusion Puzzle). Now, Matt Landreman at the University of Maryland, College Park, and Elizabeth Paul at Princeton University have given a boost to an alternative kind of fusion reactor called a stellarator. Using a numerical model, the researchers show that a particular magnetic-field arrangement, referred to as

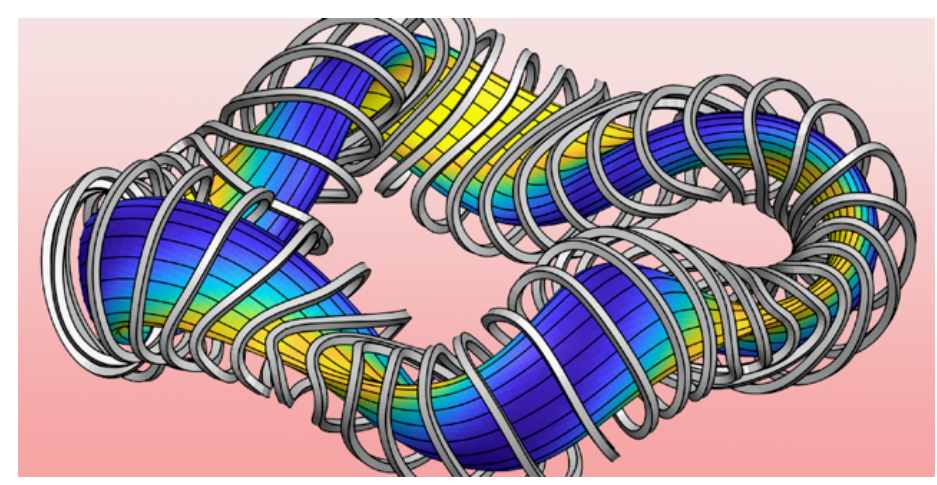

Figure 1: A quasisymmetric magnetic field (colored surface) and the electromagnetic coils (grey rings) that generate the field-line rotational transform.

Credit: M. Landreman/University of Maryland quasisymmetry, which allows long-term plasma confinement, can be achieved in a stellarator much more precisely than previously thought [2].

The idea behind magnetic confinement in both tokamaks and stellarators is to create, using an overall toroidal geometry, a system with closed field lines along which charged particles can cycle endlessly. However, while charged particles follow these magnetic-field lines for the most part, they can still drift away and escape. Moreover, positive ions and electrons drift in different directions, leading to charge separation and to a consequent electric field that can push charged particles away from the field lines. One solution to this problem is to apply a "field-line rotational transform," a magnetic-field configuration in which the field lines are twisted poloidally around the torus to form magnetic surfaces. Restricting the charged-particle motion to these surfaces suppresses the possibility of building up electric fields in the system.

The method used to generate the field-line rotational transform is the main difference between the tokamak and stellarator concepts. In a tokamak, the field-line rotational transform is created by having the torus encircle one side of a transformer, thereby inducing a toroidal current in the plasma [3]. An important advantage of this design is that its simple geometry leads to a magnetic field with a toroidal symmetry in both its amplitude and its vector. This symmetry improves long-term confinement by promoting "particle motion invariance"-the particles follow closed orbits that don't deviate much from their respective nested magnetic surfaces. The drawback of the design is that it requires a direct toroidal plasma current instead of an alternating one. However, a continuously increasing current would be needed to generate a direct current in the plasma torus. Since such a continuously increasing current 
cannot be maintained, it was realized from day one that saturation of the induced current in a tokamak would present a challenge to long-term plasma confinement. Although various alternative current-drive schemes are being considered, steady-state tokamak operation remains difficult to achieve.

A stellarator avoids this problem by generating the field-line through a series of twisted coils, which wrap around the outside of the torus (Fig. 1) [4]. However, this design has a drawback because it lacks the toroidal symmetry of the tokamak. Consequently, the existence of nested magnetic surfaces cannot be guaranteed and, more generally, trapped particles (those that are bounced back and forth by the inhomogeneous magnetic field, instead of circulating along the field lines) can still drift away from the system even with good magnetic surfaces. Particle motion invariance can help to confine these trapped particles, but it needs some kind of tokamak-like symmetry. It is this consideration that motivates the research of quasisymmetric stellarators.

The concept of quasisymmetry relies on the fact that systems with nested magnetic surfaces can be described using so-called Boozer coordinates [5]. In this coordinate system, the scalar magnitude of the magnetic field vector can be defined by specifying two directions on a magnetic surface: the field's poloidal and toroidal angles ( $\theta$ and $\varphi$, respectively).

Quasisymmetry is achieved, that is, the scalar magnitude of the magnetic field comes to depend only on a single angle-like variable on a magnetic surface, when the magnetic field is constructed such that the field strength $\mathrm{B}=\mathrm{B}(\Psi, M \theta-N \varphi)$, where $\Psi$ is the magnetic surface, and $M$ and $N$ specify the numbers of periods in the poloidal and toroidal directions, respectively. Under these conditions, particle motion invariance is regained, and plasma confinement is improved [6]. Stellarator configurations that approximate such quasisymmetry have been obtained numerically [7], but until now, precise quasisymmetry has remained elusive. It has even been suggested that precise quasisymmetry could not be achieved beyond an infinitesimally thin volume. The task is so difficult because the plasma column must be shaped and twisted (to generate the field-line rotational transform), yet the scalar magnitude of the magnetic field is required to have a symmetric direction on the magnetic surfaces.

Previous numerical attempts to achieve quasisymmetry have mainly been based on minimizing the symmetry-breaking Fourier components of the scalar amplitude of the magnetic field in Boozer coordinates at each iteration. Landreman and Paul approach the problem by numerically minimizing an objective function that includes the toroidal and poloidal currents inside a magnetic surface [2]. Their result is a breakthrough because they find certain parameter combinations that achieve quasisymmetry more precisely than any previous efforts. The improvement is substantial, reducing the deviation from quasisymmetry on a magnetic surface by more than an order of magnitude and achieving good symmetry throughout the whole plasma volume.

As in most quasisymmetric stellarator configurations, Landreman and Paul employ a field-line rotational transform that is roughly constant from the center to the edge of the plasma volume. They choose large values of $M$ and $N$ in order to avoid the creation of resonance surfaces with small rational numbers, which potentially lead to the appearance of large magnetic islands. Such islands break the nested-magnetic-surface structure and can weaken the plasma confinement. The duo hasn't yet considered configurations with finite magnetic shear, which can improve plasma stability. But their numerical scheme could also be useful in this further effort.

As important as this breakthrough is, a theoretical demonstration of quasisymmetry is only one step toward a working stellarator. In order to realize quasisymmetrical confinement, researchers need to construct the appropriate system of current-carrying coils. Numerical methods that can be used to design such systems have been established [8], but physical experiments can include considerable errors, which may well exceed the magnitude of the error achieved by Landreman and Paul. The good news, as the duo points out, is that the challenge of engineering sufficiently accurate magnetic fields might be simplified using permanent magnets in addition to electrical coils [9].

Linjin Zheng: Institute for Fusion Studies, The University of Texas at Austin, Austin, TX

\section{REFERENCES}

1. K. Ikeda, "Progress in the ITER physics basis," Nucl. Fusion 47 (2007). 
2. M. Landreman and E. Paul, "Magnetic fields with precise quasisymmetry for plasma confinement," Phys. Rev. Lett. 128, 035001 (2022).

3. L. A. Artsimovich, "Research into controlled thermonuclear reactions in the USSR," Sov. J. At. Energy 5, 1411 (1958).

4. L. A. Spitzer, "Proposed stellarator," Technical Report, Project Matterhorn (1951).

5. A. H. Boozer, "Establishment of magnetic coordinates for a given magnetic field," Phys. Fluids 25, 520 (1982).
6. A. H. Boozer, "Transport and isomorphic equilibria," Phys. Fluids 26, 496 (1983).

7. J. Nührenberg and R. Zille, "Quasi-helically symmetric toroidal stellarators," Phys. Lett. A 129, 113 (1988).

8. P. Merkel, "Solution of stellarator boundary value problems with external currents," Nucl. Fusion 27, 867 (1987).

9. P. Helander et al., "Stellarators with Permanent Magnets," Phys. Rev. Lett. 124, 095001 (2020). 